\title{
Single approximation for Biobjective Max TSP*广
}

\author{
Cristina Bazgan ${ }^{1,2,3}$ \\ Laurent Gourvès ${ }^{2,1}$ \\ Jérôme Monnot ${ }^{2,1}$ \\ Fanny Pascual ${ }^{4}$ \\ 1. Université Paris-Dauphine, LAMSADE, \\ Place du Maréchal de Lattre de Tassigny, 75775 Paris Cedex 16, France \\ 2. CNRS, UMR 7243 \\ 3. Institut Universitaire de France \\ 4. Université Pierre et Marie Curie, LIP6, 4 place Jussieu, 75005 Paris, France \\ \{bazgan, laurent.gourves, monnot\}@lamsade.dauphine.fr, fanny.pascual@lip6.fr
}

\begin{abstract}
We mainly study Max TSP with two objective functions. We propose an algorithm which returns a single Hamiltonian cycle with performance guarantee on both objectives. The algorithm is analysed in three cases. When both (resp. at least one) objective function(s) fulfill(s) the triangle inequality, the approximation ratio is $\frac{5}{12}-\varepsilon \approx 0.41$ (resp. $\frac{3}{8}-\varepsilon$ ). When the triangle inequality is not assumed on any objective function, the algorithm is $\frac{1+2 \sqrt{2}}{14}-\varepsilon \approx 0.27$-approximate.
\end{abstract}

\section{Introduction}

The traveling salesman problem (TSP) is one of the most studied problems in combinatorial optimization. Given an undirected complete graph with weights on the edges, the problem consists of finding a Hamiltonian cycle (also called tour) of maximum or minimum total weight, defined as the sum of its edges' weights. In this paper we mainly study the approximation of the biobjective maximization version, Biobjective Max TSP. In this case every edge has two weights and the total weight of a tour is a couple defined as the componentwise sum of its edges' weights. We are interested in the existence and the computation in polynomial time of a single tour with simultaneous performance guarantees on the two objectives. Our work falls into a recent stream of research on the approximability of multiobjective optimization problems $[22,21,19,10,5,12,3,1,6]$ where multiobjective TSP takes a prominent place $[8,2,4,17,7,11,15,16]$.

In many real optimization problems not only one objective function is considered but several ones (see [9] about multiobjective combinatorial optimization). This is also the case for TSP where we might want to minimize the travel time, the cost or to maximize the profit, the number of viewpoints along the way etc. This gives rise to Multiobjective TSP. Unfortunately it is unlikely that optimality is met simultaneously by a single feasible solution on all objectives. However there always exists a set of efficient (also called Pareto optimal)

\footnotetext{
${ }^{*}$ Supported by ANR-09-BLAN-0361 GUaranteed Efficiency for PAReto optimal solutions Determination (GUEPARD)

${ }^{\dagger}$ A preliminary version appears in the proceedings of WAOA 2011.
} 
solutions for which any improvement on one objective induces a deterioration of (at least) another one.

Generating the whole set of efficient solutions is a major challenge in multiobjective combinatorial optimization. However, even for moderately-sized problems, it is usually computationally prohibitive to identify the efficient set for two major reasons. First, the number of efficient solutions can be very large. Second, the associated decision version is often NPcomplete, even if the underlying single objective problem is polynomial time solvable. To handle these two difficulties, researchers have been interested in developing approximation algorithms with a priori provable performance guarantees.

Given a positive real $\rho \leq 1$, and considering that all objectives have to be maximized, a $\rho$-approximation of the set of efficient solutions is a set of solutions that includes, for each efficient solution, a solution that approximates it within a factor $\rho$ on all objectives. The $\rho$-approximation typically contains several incomparable solutions and it is assumed that one solution is selected with the help of a, yet unkown, a posteriori decision process.

One of the most important results concerning the approximation of multiobjective problems was given by Papadimitriou and Yannakakis [19]: under certain general assumptions, multiobjective optimization problems always have a $(1-\varepsilon)$-approximation of size polynomial in the size of the instance and $1 / \varepsilon$, for any given accuracy $\varepsilon>0$. This result makes the computation of approximate efficient sets of multiobjective problems accessible to polynomial time algorithms.

Nevertheless the efficient set is not the unique object that one can approximate. A popular approach in multiobjective optimization consists in optimizing only one objective while the others are turned into budget constraints $[22,21,12,6]$. Budget constraints come from an $a$ priori decision process which restricts the set of desired solutions. It is noteworthy that the efficient set approach and the budget approach are essentially the same [19].

In another popular approach, no decision process is sought. The goal is to compute a single solution which approximates a vector composed of the optimal values on every objective taken separately $[23,8,20,3,1]$. Contrasting with the previous approaches, this framework aims at approximating an ideal point which is the image of a not necessarily feasible solution. Hence $\rho$-approximations are not guaranteed to exist for all $\rho$. Note that the ideal point approach and the efficient set approach restricted to sets of size 1 coincide. The former is a particular case of the latter. Since generating several solutions allows better approximations than what a single solution can achieve, approximation ratios under the respective approaches are not directly comparable.

Previous results for the multiobjective TSP are known; most of them follow the efficient set approach, approximating the Pareto set with two or more solutions, but some of them use the ideal point approach. In this article we exclusively follow the ideal point approach and provide deterministic approximation algorithms whose performance guarantees improve on previous results.

Previous results. Multiobjective TSP has been well studied from the approximation point of view.

Manthey and Ram [17] have followed the efficient set approach for several variants of multiobjective Min TSP. In particular they have generalized the well known tree doubling algorithm to provide a $(2+\epsilon)$-approximation of the efficient set (see also [8] for an earlier tree doubling algorithm). The other results of [17] deal with multiobjective Min TSP with the sharpened triangle inequality and multiobjective Min TSP with distance 1 or 2 . This latter 
problem has been studied in $[2,4]$ under the efficient set approach.

More rencently Bläser et al. [7] have studied the multiobjective Max TSP with $k$ objective functions. Using the efficient set approach they have devised randomized approximation algorithms with ratios $\frac{1}{k}-\epsilon$ and $\frac{1}{k+1}-\epsilon$ for the symmetric and asymmetric versions respectively. Subsequently these results have been significantly improved by Manthey [16] who has provided randomized approximation algorithms, using the efficient set approach, with ratios $\frac{2}{3}-\epsilon$ and $\frac{1}{2}-\epsilon$ for the symmetric and asymmetric versions respectively. These algorithms use as a black box the randomized PTAS for min-weight matching given by Papadimitriou and Yannakakis [19]. Glaßer, Reitwießner and Witek have slightly improved the ratios to $\frac{2}{3}$ and $\frac{1}{2}$, respectively [11]. Recently, Manthey [14] has established deterministic approximation algorithms, using the efficient set approach, with ratios $\frac{1}{2 k}-\epsilon$ and $\frac{1}{4 k-2}-\epsilon$ for the symmetric and asymmetric versions respectively that can be improved for the biobjective case to ratios $\frac{3}{8}-\epsilon$ and $\frac{1}{4}-\epsilon$ respectively.

Manthey has also investigated the approximation of Biobjective Max TSP under the ideal point approach [16, 14], i.e. approximate efficient sets of size one. If the single objective Max TSP problem is $\rho$-approximable then Biobjective Max TSP is $\frac{\rho}{3}$-approximable with one solution [16]. Taking the best polynomial time approximation algorithms known so far for the symmetric Max TSP, he has derived a $\frac{7}{27}$-approximate (resp. $\frac{7}{24}$-approximate) tour without (resp. with) the triangle inequality.

Another positive consequence of the general technique is that every biobjective instance admits a single $\frac{1}{3}$-approximate tour. From the negative side, Manthey [16] has given a 5 node non metric instance in which no single tour can be $(1 / 3+\epsilon)$-approximate $(\epsilon>0)$, thus meeting the previous bound. To our best knowledge, no such upper bound is known for metric instances so it is still possible that a single $\rho$-approximate tour exists in biobjective Max TSP for some $\rho>1 / 3$. Finally one can observe that known inapproximability results on the single objective Max TSP imply that the general technique is limited to provide biobjective $(1 / 3-\epsilon)$-approximation in polynomial time $(\epsilon>0)$.

New results. In this paper, we establish a general algorithm which computes a maximum value matching on each objective taken separately and combines them into a single Hamiltonian cycle having a performance guarantee on both objectives. The algorithm is analyzed in three cases. When both objective functions fulfill the triangle inequality, we obtain a $\frac{5}{12}-\epsilon \approx 0.41$-approximate algorithm which improves the aforementioned $\frac{7}{24}-\epsilon \approx 0.291$ approximation. In this case, we also propose a 4-node instance without any single $\left(\frac{1}{2}+\epsilon\right)$ approximate solution and a family of instances without any single $\left(\frac{3}{4}+\epsilon\right)$-approximate solution when the number of nodes tends to infinity. If only one objective function fulfills the triangle inequality, we obtain a $\left(\frac{3}{8}-\epsilon\right)$-approximate algorithm. In the case where no objective function satisfies the triangle inequality, a quick analysis gives a ratio $1 / 4-\epsilon$ but in a more accurate case analysis, we can show that the algorithm is $\frac{1+2 \sqrt{2}}{14}-\epsilon \approx 0.27$-approximate, improving the aforementioned $\frac{7}{27} \approx 0.259$-approximation. An extension of Manthey's instance to any number of vertices precludes any $\left(\frac{1}{3}+\epsilon\right)$-approximate algorithm returning one solution.

Table 1 gives a summary of mentioned results on the biobjective Max TSP $(k=2)$. Approximations achieved with several solutions follow the Pareto set approach while those limited to one solution follow the ideal point approach.

Organization of the article. In Section 2 we give definitions on the problems and concepts used throughout the article. In Section 3 we establish some non existence results which 


\begin{tabular}{|c|c|c|c|}
\hline \multicolumn{3}{|c|}{ Biobjective Max TSP } \\
\hline & randomized algo. & deterministic algo. & this paper $($ deterministic $)$ \\
\hline general & $2 / 3[11]$ & $7 / 27 \approx 0.259$ & $\frac{1+2 \sqrt{2}}{14}-\epsilon \approx 0.27$ \\
case & several solutions & one solution $[16,18]$ & $3 / 8-\epsilon$ \\
& & several solutions $[14]$ & \\
& & $7 / 24 \approx 0.291$ & $5 / 12-\epsilon \approx 0.41$ \\
metric & $2 / 3[11]$ & one solution $[16,13]$ & one solution \\
case & several solutions & one solut \\
\hline
\end{tabular}

Table 1: Approximation results for Biobjective MaxTSP

give upper bounds on possible approximation ratios under the ideal point approach. Section 4 presents a general algorithm for Biobjective Max TSP and its analysis in three cases depending on the (non) metric nature of the objective functions. In Section 5 we improve the analysis of the previous algorithm in the non metric case.

\section{Preliminaries}

Let $G=(V, E)$ be a complete undirected graph with a nonnegative weight $w(e)$ on every edge $e \in E$ and $n=|V|$ vertices. The weight of a set of edges $E^{\prime} \subseteq E$ is the sum of the weights of the edges in $E^{\prime}$ and is denoted by $w\left(E^{\prime}\right)$. An instance is metric if its weights satisfy the triangle inequality, namely $w(x, z) \leq w(x, y)+w(y, z)$ for all distinct vertices $x, y, z \in V$.

Max TSP is to find a Hamiltonian cycle or tour (i.e. a cycle that visits every vertex of the graph exactly once) of maximum weight in a complete graph. In the multiobjective Maximum Traveling Salesman Problem every edge is endowed with $k$ nonnegative values. For the biobjective case $(k=2)$, each edge $e \in E$ has a nonnegative weight $w(e)$ and a nonnegative length $\ell(e)$. Similarly the length of a set of edges $E^{\prime}$, denoted by $\ell\left(E^{\prime}\right)$, is the sum of the lengths of its elements.

Each feasible tour $T$ is represented in the objective space by its corresponding objective vector $(w(T), \ell(T))$. A tour $T$ dominates a tour $T^{\prime}$ if and only if $w(T) \geq w\left(T^{\prime}\right)$ and $\ell(T) \geq$ $\ell\left(T^{\prime}\right)$ with at least one strict inequality. A tour $T$ is efficient if and only if no other tour $T^{\prime}$ dominates $T$, and $(w(T), \ell(T))$ is said to be non-dominated. An efficient set contains, for each non-dominated vector, a corresponding efficient solution (no need to keep two tours having the same objective vector).

Unfortunately computing the efficient set of multiobjective Max TSP cannot be done in polynomial time, unless $P=N P$, so we are interested in its polynomial time computable approximations. For any $0<\rho \leq 1$, a tour $T \rho$-approximates another tour $T^{*}$ if and only if $w(T) \geq \rho w\left(T^{*}\right)$ and $\ell(T) \geq \rho \ell\left(T^{*}\right)$. A set of feasible tours $\mathcal{A}$ is a $\rho$-approximation of the efficient set $\mathcal{P}$ if for every $T^{*} \in \mathcal{P}$, there exists $T \in \mathcal{A}$ such that $T \rho$-approximates $T^{*}$. If $\mathcal{A}$ is reduced to a single tour, we say that we follow the ideal point approach.

Define opt $_{w}\left(\right.$ resp. opt $t_{\ell}$ ) as $\max _{T \in \mathcal{F}} w(T)$ (resp. $\max _{T \in \mathcal{F}} \ell(T)$ ) where $\mathcal{F}$ denotes the set of feasible tours. Under the ideal point approach, a tour $T$ is a $\rho$-approximation if and only if $w(T) \geq \rho$ opt $_{w}$ and $\ell(T) \geq \rho$ opt $_{\ell}$. 


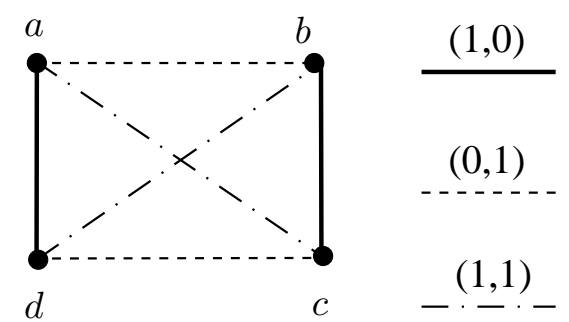

Figure 1: There is no $(0.5+\epsilon)$-approximate solution in this instance where every objective function satisfies the triangle inequality.

\section{Non existence of a single $\rho$-approximate solution}

It is unlikely that every instance admits a single solution which is nearly optimal for $w$ and $\ell$ at the same time. Thus instances without any $\rho$-approximate solution imply that no deterministic $\rho$-approximate algorithm (even exponential) exists.

If the triangle inequality is satisfied on both objectives, the example given in Figure 1 shows that there does not always exist a $\left(\frac{1}{2}+\epsilon\right)$-approximate solution, for all $\epsilon>0$. The three possible tours in this instance are indeed $(a, b, c, d, a),(a, c, d, b, a)$, and $(a, c, b, d, a)$ whose values are $(2,2),(2,4)$, and $(4,2)$. However this instance only contains 4 nodes so it does not prevent an algorithm to provide a $(0.5+\epsilon)$-approximate solution for 5 nodes and more. On the positive side, if the graph contains 4 nodes then a $1 / 2$-approximate tour must exist (no need to impose the triangle inequality). Indeed the union of two matchings form a matching or a tour. Then the union of a maximum weight matching and a maximum length matching gives a tour which is 1/2-approximate, or a matching which can be completed without any deterioration.

However one can build an instance which does not contain any $\left(\frac{2}{3}+\epsilon\right)$-approximate solution for $n$ sufficiently large. Consider a complete graph on $2 r$ vertices $G=(V, E)$ where $V=V_{1} \cup V_{2}$ and $\left|V_{1}\right|=\left|V_{2}\right|=r$. The edges with their two endpoints in the same part of the bipartition have weight 2 and length 0 . The other edges have weight and length 1 . It is not difficult to see that the triangle inequality is satisfied on both the weight and the length. An optimal weight tour has value $4 r-2$ and an optimal length tour has value $2 r$. Consider a tour $T$ which contains $d$ edges with value $(1,1)$ and $2 r-d$ edges with value $(2,0)$. Then $T$ has value $(4 r-d, d)$. Now observe that $\min \left\{\frac{4 r-d}{4 r-2}, \frac{d}{2 r}\right\} \leq \frac{2}{3-1 / r}$, for all $d$. As $r$ grows, we cannot expect a single tour to be $\left(\frac{2}{3}+\varepsilon\right)$-approximate on both objectives, where $\varepsilon$ is a positive constant.

If the objective functions do not necessarily fulfill the triangle inequality, Manthey [16] proved that for a $K_{5}$ there does not exist a $\left(\frac{1}{3}+\epsilon\right)$-approximate algorithm, for all $\epsilon>0$. We can easily generalize his result to $K_{n}$ with $n \geq 5$ in order to obtain an asymptotic result. For every $n \geq 5$, consider $K_{n}$ where one fixed $K_{4}$ is decomposed into two Hamiltonian paths $P_{w}$ and $P_{\ell}$. For every edge $e \in E\left(K_{n}\right)$, set $w(e)=1$ and $\ell(e)=0$ if $e \in P_{w}, w(e)=0$ and $\ell(e)=1$ if $e \in P_{\ell}$ and $w(e)=0$ and $\ell(e)=0$ if $e \notin P_{w} \cup P_{\ell}$ (every edge outside the fixed $K_{4}$ has weight and length equal to 0$)$. We can check that there are four non-dominated tours $T_{i}, i=1, \ldots, 4$ with $w\left(T_{1}\right)=w\left(P_{w}\right)=3, \ell\left(T_{1}\right)=\ell\left(P_{w}\right)=0, w\left(T_{2}\right)=w\left(P_{\ell}\right)=0$, $\ell\left(T_{2}\right)=\ell\left(P_{\ell}\right)=3, w\left(T_{3}\right)=2, \ell\left(T_{3}\right)=1$ and $w\left(T_{4}\right)=1, \ell\left(T_{4}\right)=2$. In conclusion, a single solution never approximates the Pareto set of the biobjective Max TSP with ratio better than 
$1 / 3$ for $K_{n}$ with $n \geq 5$.

\section{A generic algorithm for Biobjective Max TSP}

In this section, we present an algorithm for the Biobjective Max TSP. This algorithm is based on the combination of the edges of a maximum weight matching for the objective $w$ and a maximum weight matching for the objective $\ell$. Recall that the values on the edges are non negative so adding an edge to a partial solution cannot deteriorate its weight or length. The algorithm is as follows :

1. Build a maximum weight (resp. length) matching of $G$ and denote it by $M_{w}$ (resp. $M_{\ell}$ ). The set of edges $M_{w} \cup M_{\ell}$ is made of $p$ connected components $C_{1}, \ldots, C_{p}$. Each $C_{i}$ is a cycle of even size, or a path of length at least one. Note that there is at most one path of length at least two in $M_{w} \cup M_{\ell}$ (because the graph is complete and we can assume that $M_{w}$ are $M_{\ell}$ are of maximum size). Likewise, each path of length one is in $M_{w} \cap M_{\ell}$.

2. For each component $C_{i}$ which is a cycle, remove the edge in $C_{i} \cap M_{w}$ which has minimum weight.

We thus obtain a set of paths, which is called a partial tour.

3. Add edges in order to connect these paths and obtain an Hamiltonian cycle of $K_{n}$ (edges are added arbitrarily unless otherwise noted. This step is detailled inside the proofs when needed).

Let us now show that the Hamiltonian cycle obtained with this algorithm has a weight larger than or equal to $\alpha w\left(M_{w}\right)$ and a length larger than or equal to $\alpha \ell\left(M_{\ell}\right)$, where $0<\alpha \leq 1$. We will determine the value of $\alpha$ in a general graph (cf. Lemma 1), in a graph where one objective function (w.l.o.g. $w$ ) fulfills the triangle inequality (cf. Lemma 2), and in a graph where both objective functions fulfill the triangle inequality (cf. Lemma 3).

Lemma 1 Step 1 and 2 of the algorithm build in polynomial time a partial tour on $K_{n}$ with weight at least $\frac{1}{2} w\left(M_{w}\right)$ and length at least $\frac{1}{2} \ell\left(M_{\ell}\right)$.

Proof : For each component $C_{i}$ which is a cycle, step 2 of the algorithm removes the edge in $C_{i} \cap M_{w}$ with minimum weight. Since $\left|C_{i} \cap M_{w}\right| \geq 2$ the loss in weight is at most $w\left(C_{i} \cap M_{w}\right) / 2$. The resulting set of edges is a partial tour of weight at least $\frac{1}{2} \sum_{i=1}^{p} w\left(C_{i} \cap M_{w}\right)=\frac{1}{2} w\left(M_{w}\right)$ and length $\sum_{i=1}^{p} \ell\left(C_{i} \cap M_{\ell}\right)=\ell\left(M_{\ell}\right)$.

In the following Lemmas we consider two cases:

- Case 1: at the end of Step 1 of the algorithm, every component $C_{i}$ is a cycle

- Case 2: at the end of Step 1 of the algorithm, at least one component $C_{i}$ is a cycle and at least one component $C_{i^{\prime}}$ is not a cycle.

If no component is a cycle then we are already done since the set of edges is then a partial tour of weight $w\left(M_{w}\right)$ and length $\ell\left(M_{\ell}\right)$.

Lemma 2 Assuming that $w$ satisfies the triangle inequality, we can build in polynomial time a partial tour on $K_{n}$ with weight at least $\frac{3}{4} w\left(M_{w}\right)$ and length at least $\frac{3}{4} \ell\left(M_{\ell}\right)$. 
Proof : We distinguish two cases depending on the value of $p$ that is the number of connected components of $M_{w} \cup M_{\ell}$. If $p=1$ then $C_{1}$ is either a tour or a cycle on $n-1$ nodes (in this case $n$ is odd) with weight at least $w\left(M_{w}\right)$ and length at least $\ell\left(M_{\ell}\right)$. If $C_{1}$ is a cycle on $n-1$ nodes, let $x$ be the isolated node. Then by replacing any edge $(u, v) \in M_{w}$ by $(u, x),(x, v)$, we get a tour $C^{\prime}$ of $K_{n}$ satisfying $w\left(C^{\prime}\right) \geq w\left(C_{1}\right) \geq w\left(M_{w}\right)$ due to the triangle inequality and $\ell\left(C^{\prime}\right) \geq \ell\left(M_{\ell}\right)$.

Let us now consider the case where $p \geq 2$. Assume that case 1 occurs, that is each component $C_{i}$ is a cycle and thus it contains at least four edges. Since $p \geq 2$ and $\left|M_{\ell} \cap C_{i}\right| \geq 2$ for each $C_{i}$ we have $\left|M_{\ell}\right| \geq 4$. It follows that if $e \in M_{\ell}$ is an edge of minimum length among the edges of $M_{\ell}$, then $\ell(e) \leq \ell\left(M_{\ell}\right) / 4$. Thus, by deleting $e$, we are in case 2 since $\cup_{i=1}^{p} C_{i} \backslash\{e\}$ contains at least one cycle and at least one path with $w\left(\cup_{i=1}^{p} C_{i} \backslash\{e\}\right) \geq w\left(M_{w}\right)$ and

$$
\ell\left(\cup_{i=1}^{p} C_{i} \backslash\{e\}\right) \geq 3 \ell\left(M_{\ell}\right) / 4
$$

Now, assume that case 2 occurs. By renaming the connected components, we can assume that there is an integer $r \in\{1, \ldots, p\}$ such that $C_{i}$ for $i \geq r$ is not a cycle whereas $C_{i}$ for $1 \leq i<r$ is a cycle. Let $x$ and $y$ be the two extremities of $C_{r}$. Proceed repeatedly as follows, for $i=r-1$ down to 1 . Remove an edge of minimum weight in $M_{w} \cap C_{i}$ and call it $\left(v_{1}^{i}, v_{2}^{i}\right)$. If $w\left(v_{1}^{i}, x\right) \geq w\left(v_{2}^{i}, x\right)$ then add the edge $\left(v_{1}^{i}, x\right)$ and set $x:=v_{2}^{i}$, otherwise add the edge $\left(v_{2}^{i}, x\right)$ and set $x:=v_{1}^{i}$. By this way the procedure maintains a path with extremities $x$ and $y$, while reducing the number of cycles. At the end of the procedure we get a partial tour that is the union of a path and $\cup_{i=r}^{p} C_{i}$. Using the triangle inequality we know that $\max \left\{w\left(v_{1}^{i}, x\right), w\left(v_{2}^{i}, x\right)\right\} \geq\left(w\left(v_{1}^{i}, x\right)+w\left(v_{2}^{i}, x\right)\right) / 2 \geq w\left(v_{1}^{i}, v_{2}^{i}\right) / 2$, meaning that each time an edge $\left(v_{1}^{i}, v_{2}^{i}\right)$ is removed $(i \in\{1, \ldots, r-1\})$, another one with at least half its weight is added so, in total, the loss in weight is bounded by $\frac{1}{2} \sum_{i=1}^{r-1} w\left(v_{1}^{i}, v_{2}^{i}\right)$. Since $\left|M_{w} \cap C_{i}\right| \geq 2$ we deduce that $w\left(v_{1}^{i}, v_{2}^{i}\right) \leq w\left(M_{w} \cap C_{i}\right) / 2$. Summing up the previous inequality, we deduce that $\sum_{i=1}^{r-1} w\left(v_{1}^{i}, v_{2}^{i}\right) \leq w\left(\cup_{i=1}^{r-1} C_{i} \cap M_{w}\right) / 2 \leq w\left(M_{w}\right) / 2$. Thus the total loss in weight is bounded by $w\left(M_{w}\right) / 4$.

In conclusion the partial tour has weight at least $3 w\left(M_{w}\right) / 4$ and length at least $3 \ell\left(M_{\ell}\right) / 4$ by inequality (1).

Lemma 3 Assuming that $w$ and $\ell$ satisfy the triangle inequality, we can build in polynomial time a partial tour on $K_{n}$ with weight at least $\frac{5}{6} w\left(M_{w}\right)$ and length at least $\left(\frac{5}{6}-\varepsilon(n)\right) \ell\left(M_{\ell}\right)$. Here $\varepsilon(n)=2 /(n-1)$ and then tends to 0 when $n$ tends to $\infty$.

Proof: As it is done in Lemma 2, we transform case 1 into case 2. Thus, suppose that we are in case 1 that is each component $C_{i}$ is a cycle and w.l.o.g. that the edge of $M_{\ell}$ with minimum length is $e$. Remove this edge $e$ to create a path with endpoints denoted by $x$ and $y$. When $n$ is even (resp. odd) this deletion induces a loss of at most $2 \ell\left(M_{\ell}\right) / n=\varepsilon(n) \ell\left(M_{\ell}\right)$ (resp. $\left.2 \ell\left(M_{\ell}\right) /(n-1)=\varepsilon(n) \ell\left(M_{\ell}\right)\right)$. Note that $\varepsilon(n)$ tends to 0 when $n$ tends to $\infty$.

Suppose now that we are in the case 2 . As it is done in Lemma 2 we can assume that there is an integer $r \in\{1, \ldots, p\}$ such that $C_{i}$ for $i \geq r$ is not a cycle whereas $C_{i}$ for $1 \leq i<r$ is a cycle. We are going to patch the cycles to $C_{r}$, one by one. We explain how to patch $C_{1}$, and the procedure is repeated for the cycles $C_{2}, \cdots, C_{r-1}$. Let $x$ and $y$ be the two extremities of $C_{r}$.

If $\left|C_{1} \cap M_{w}\right| \geq 3$ then delete an edge of minimum weight and call it $\left(v_{1}^{1}, v_{2}^{1}\right)$. We get that $w\left(v_{1}^{1}, v_{2}^{1}\right) \leq \frac{1}{3} w\left(C_{1} \cap M_{w}\right)$. Add the edge with maximum weight between $\left(v_{1}^{1}, x\right)$ and $\left(v_{2}^{1}, x\right)$. 
By the triangle inequality, $\max \left\{w\left(v_{1}^{1}, x\right), w\left(v_{2}^{1}, x\right)\right\} \geq w\left(v_{1}^{1}, v_{2}^{1}\right) / 2$. If $w\left(v_{1}^{1}, x\right) \geq w\left(v_{2}^{1}, x\right)$ then $x:=v_{2}^{1}$, otherwise $x:=v_{1}^{1}$. Disregarding the weight of the edges in $C_{1} \cap M_{\ell}$, the modification causes a loss in weight of at most $w\left(v_{1}^{1}, v_{2}^{1}\right)-w\left(v_{1}^{1}, v_{2}^{1}\right) / 2=w\left(v_{1}^{1}, v_{2}^{1}\right) / 2 \leq \frac{1}{6} w\left(C_{1} \cap M_{w}\right)$. Since no edge from $M_{\ell}$ was removed, and disregarding the length of the edges in $C_{1} \cap M_{w}$, the modification does not cause any loss in length. Hence the patching guarantees that the new path $P$ satisfies $w(P) \geq w\left(C_{r}\right)+5 w\left(C_{1} \cap M_{w}\right) / 6$ and $\ell(P) \geq \ell\left(C_{r}\right)+\ell\left(C_{1} \cap M_{\ell}\right)$.

Now suppose that $C_{1}$ is a cycle on 4 nodes and contains four edges $(a, b),(b, c),(c, d)$, $(d, a)$ such that $C_{1} \cap M_{w}=\{(a, b),(c, d)\}$ and $C_{1} \cap M_{\ell}=\{(b, c),(a, d)\}$. Using the triangle inequality we get that

$$
\begin{aligned}
w(a, c)+w(b, d)+w\left(C_{1} \cap M_{\ell}\right) & \geq w\left(C_{1} \cap M_{w}\right) \\
\ell(a, c)+\ell(b, d)+\ell\left(C_{1} \cap M_{w}\right) & \geq \ell\left(C_{1} \cap M_{\ell}\right)
\end{aligned}
$$

- Suppose that $\ell\left(C_{1} \cap M_{w}\right) \geq \ell\left(C_{1} \cap M_{\ell}\right) / 8$. W.l.o.g., assume $\ell(a, d) \geq \ell(b, c)$. Remove $(b, c)$ and add the edge with maximum length between $(b, x)$ and $(x, c)$. Since $\max \{\ell(b, x), \ell(x, c)\} \geq \ell(b, c) / 2$ by the triangle inequality, we get that the new path $P$ satisfies $\ell(P) \geq \ell\left(C_{r}\right)+\ell\left(C_{1} \cap M_{w}\right)+\ell(a, d)+\ell(b, c) / 2 \geq \ell\left(C_{r}\right)+\ell\left(C_{1} \cap M_{\ell}\right) / 8+$ $\ell\left(C_{1} \cap M_{\ell}\right) / 2+\ell(a, d) / 2 \geq \ell\left(C_{r}\right)+\ell\left(C_{1} \cap M_{\ell}\right) / 8+\ell\left(C_{1} \cap M_{\ell}\right) / 2+\ell\left(C_{1} \cap M_{\ell}\right) / 4=$ $\ell\left(C_{r}\right)+7 \ell\left(C_{1} \cap M_{\ell}\right) / 8$.

- Suppose that $w\left(C_{1} \cap M_{\ell}\right) \geq w\left(C_{1} \cap M_{w}\right) / 8$. W.l.o.g., assume $w(a, b) \geq w(c, d)$. Remove $(c, d)$ and add the edge with maximum length between $(c, x)$ and $(x, d)$. Since $\max \{w(c, x), w(x, d)\} \geq w(c, d) / 2$ by the triangle inequality, we get as in the previous case that $w(P) \geq w\left(C_{r}\right)+w\left(C_{1} \cap M_{\ell}\right)+w(a, b)+w(c, d) / 2 \geq w\left(C_{r}\right)+7 w\left(C_{1} \cap M_{w}\right) / 8$.

- Now suppose that $\ell\left(C_{1} \cap M_{w}\right)<\ell\left(C_{1} \cap M_{\ell}\right) / 8$ and $w\left(C_{1} \cap M_{\ell}\right)<w\left(C_{1} \cap M_{w}\right) / 8$. Using Inequalities (2) and (3) we get that $w(a, c)+w(b, d)>7 w\left(C_{1} \cap M_{w}\right) / 8$ and $\ell(a, c)+\ell(b, d)>7 \ell\left(C_{1} \cap M_{\ell}\right) / 8$. In this case the new path $P$ obtained by adding any two edges to $(a, c),(b, d)$ and $C_{r}$ satisfies $w(P) \geq w\left(C_{r}\right)+7 w\left(C_{1} \cap M_{w}\right) / 8$ and $\ell(P) \geq \ell\left(C_{r}\right)+7 \ell\left(C_{1} \cap M_{\ell}\right) / 8$.

In conclusion, when $C_{1}$ contains four nodes, we can always patch it to $C_{r}$ so that the loss in weight (resp. length) is at most $w\left(C_{1} \cap M_{w}\right) / 8$ (resp. $\left.\ell\left(C_{1} \cap M_{\ell}\right) / 8\right)$.

We have seen that this loss is of (at most) $1 / 6$ on both objective functions when $C_{1}$ contains at least six nodes. We deduce that after the patching of all cycles $C_{i}$ for $i<r$, the current solution is a path $P$ and its weight (resp. length) is at least $w\left(C_{r}\right)+\frac{5}{6} w\left(\bigcup_{i=1}^{r-1} C_{i} \cap M_{w}\right)$ (resp. $\ell\left(C_{r}\right)+\frac{5}{6} \ell\left(\bigcup_{i=1}^{r-1} C_{i} \cap M_{\ell}\right)$ ). Adding $\cup_{i=r+1}^{p} C_{i}$ to $P$, we get a partial tour $P^{\prime}$. Using $w\left(C_{r}\right) \geq w\left(C_{r} \cap M_{w}\right)$ and $\ell\left(C_{r}\right) \geq \ell\left(C_{r} \cap M_{\ell}\right)-\varepsilon(n) \ell\left(M_{\ell}\right)$ we get that the solution $P^{\prime}$ has weight (resp. length) at least $\frac{5}{6} w\left(M_{w}\right)\left(\right.$ resp. $\left.\left(\frac{5}{6}-\varepsilon(n)\right) \ell\left(M_{\ell}\right)\right)$.

Theorem 1 We can build in polynomial time a single tour on $K_{n}$ which constitutes a $(\rho-$ $\xi(n)$ )-approximate Pareto set for the biobjective Max TSP where $\rho=5 / 12$ when $w$ and $\ell$ satisfy the triangle inequality, $\rho=3 / 8$ when only $w$ satisfies the triangle inequality and $\rho=1 / 4$ when neither $w$ nor $\ell$ satisfies the triangle inequality. Here $\xi(n)=\Theta(1 / n)$ and then tends to 0 when $n$ tends to $\infty$.

Proof: Consider first the case when $x$ and $\ell$ satisfy the triangle inequality. Lemma 3 states that we can build a partial tour with weight (resp. length) at least $5 w\left(M_{w}\right) / 6$ (resp. $\left(\frac{5}{6}-\right.$ 
$\left.\varepsilon(n)) \ell\left(M_{\ell}\right)\right)$ where $\varepsilon(n)=\frac{2}{n-1}$. If the partial tour is not a tour then connect its components to create a tour. Using the fact that every edge weight (resp. length) is nonnegative, the weight (resp. length) cannot decrease. Denote by opt $t_{w}$ (resp. opt $\ell$ ) the optimal weight (resp. length) of a tour. It is well known that $w\left(M_{w}\right) \geq\left(\frac{1}{2}-\varepsilon^{\prime}(n)\right) o p t_{w}$ and $\ell\left(M_{\ell}\right) \geq\left(\frac{1}{2}-\varepsilon^{\prime}(n)\right) o p t_{\ell}$ where $\varepsilon^{\prime}(n)=0$ when $n$ is even, otherwise $\varepsilon^{\prime}(n)=\frac{1}{2 n}$. Let $\xi(n)=\frac{\varepsilon(n)}{2}+\frac{5 \varepsilon^{\prime}(n)}{6}-\varepsilon^{\prime}(n) \varepsilon(n)$. We get that the tour constructed has weight at least $\frac{5}{6} w\left(M_{w}\right) \geq \frac{5}{6}\left(\frac{1}{2}-\varepsilon^{\prime}(n)\right) o p t_{w}>\left(\frac{5}{12}-\xi(n)\right) o p t_{w}$. The length is at least $\left(\frac{5}{6}-\varepsilon(n)\right) \ell\left(M_{\ell}\right) \geq\left(\frac{5}{6}-\varepsilon(n)\right)\left(\frac{1}{2}-\varepsilon^{\prime}(n)\right)$ opt $_{\ell}=\left(\frac{5}{12}-\xi(n)\right)$ opt $_{\ell}$. Use Lemmas 1 and 2 and similar arguments for the other cases.

\section{An improved analysis}

In this section, we refine the analysis of our approximation algorithm when the triangle inequality is not assumed on any objective function. We show that the tour returned by our algorithm is an asymptotic $\frac{1+2 \sqrt{2}}{14} \approx 0.273$ approximation of the ideal point. Recall that some instances of the problem do not admit any $\left(\frac{1}{3}+\epsilon\right)$-approximate solution, for all $\epsilon>0$ [16].

The intuition behind the improved analysis is the following. The ratio $1 / 4$ of Theorem 1 follows from two observations: the tour returned by the approximation algorithm is a $1 / 2$ approximation of the maximum weight/length matching, and this latter is an asymptotic 1/2-approximation of the maximum weight/length tour. Taken separately both observations are tight but we exploit the fact that they cannot occur simultaneously.

Next Theorem applies without requiring the triangle inequality for $w$ or $\ell$.

Theorem 2 We can build in polynomial time a $\left(\frac{1+2 \sqrt{2}}{14}-\xi(n)\right)$-approximate Pareto set containing a single tour on $K_{n}$ for Biobjective Max TSP. Here $\xi(n)=\Theta(1 / n)$ and then, tends to 0 when $n$ tends to $\infty$.

Proof: Define $\delta$ as $\frac{4 \sqrt{2}-5}{14} \approx 0.0469$. Actually, $\delta$ is the positive root of equation $-1+20 x+$ $28 x^{2}=0$. We can show that every instance $K_{n}$ of the problem satisfies one of the following statements:

(i) a partial tour $P^{\prime}$ on $K_{n}$ with weight at least $\left(\frac{1}{2}+\delta\right) w\left(M_{w}\right)$ and, at the same time, length at least $\left(\frac{1}{2}+\delta\right) \ell\left(M_{\ell}\right)$ exists and can be computed in polynomial time.

(ii) every Hamiltonian cycle has weight at most $\left(\frac{3}{2}+7 \delta\right) w\left(M_{w}\right)$ and, at the same time, its length is at most $\left(\frac{3}{2}+7 \delta\right) \ell\left(M_{\ell}\right)$.

Recall that $w\left(M_{w}\right) \geq\left(\frac{1}{2}-\varepsilon^{\prime}(n)\right) o p t_{w}, \ell\left(M_{\ell}\right) \geq\left(\frac{1}{2}-\varepsilon^{\prime}(n)\right) o p t_{\ell}$ where $\varepsilon^{\prime}(n)=0$ when $n$ is even, otherwise $\varepsilon^{\prime}(n)=1 / 2 n$. If $K_{n}$ satisfies $(i)$, then by hypothesis the partial tour $P^{\prime}$ has weight (resp. length) at least $(1 / 4+\delta / 2-\xi(n)) o p t_{w}$ (resp. $\left.(1 / 4+\delta / 2-\xi(n)) o p t_{\ell}\right)$ with $\xi(n)=\varepsilon^{\prime}(n)(1 / 2+\delta)$. If $K_{n}$ satisfies $(i i)$, then starting from $M_{w} \cup M_{\ell}$ as it is done in previous section and using Lemma 1, a partial solution $P$ with weight (resp. length) at least $w\left(M_{w}\right) / 2\left(\operatorname{resp} . \ell\left(M_{\ell}\right) / 2\right)$ can be built in polynomial time. Now, since by hypothesis opt $_{w} \leq\left(\frac{3}{2}+7 \delta\right) w\left(M_{w}\right)$, and opt $_{\ell} \leq\left(\frac{3}{2}+7 \delta\right) \ell\left(M_{\ell}\right)$, the partial solution $P$ has a weight (resp. length) at least $\frac{1}{2} \frac{o p t_{w}}{\left(\frac{3}{2}+7 \delta\right)}$ (resp. $\left.\frac{1}{2} \frac{o p t_{\ell}}{\left(\frac{3}{2}+7 \delta\right)}\right)$.

Finally remark that on the one hand, a tour can be obtained by connecting the components of a partial tour without decreasing the weight/length since every edge weight/length 


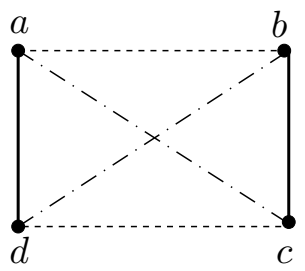

Edges of $M_{\ell}$

Edges of $M_{w}$

-.... A third matching

Figure 2: The cycle $C_{1}$. Bold edges belong to $M_{\ell}$ and dashed edges belong to $M_{w}$; the remaining edges form a third matching $M_{r}=\{(a, c),(b, d)\}$.

is nonnegative and on the other hand, $\frac{1}{2} \frac{1}{\left(\frac{3}{2}+7 \delta\right)}=1 / 4+\delta / 2=\frac{1+2 \sqrt{2}}{14}$ because $\delta$ is the positive root of equation $-1+20 x+28 x^{2}=0$.

We assume $n \geq 5$, since otherwise the partial solution $P$ given in Lemma 1 has weight (resp. length) at least $o p t_{w} / 2($ resp. opt $\ell) / 2$ ).

We consider three distinct cases which can be distinguished in polynomial time.

Case 1. Let us suppose that there exists a cycle, say $C_{1}$ w.l.o.g., such that the edge with minimum weight in $C_{1} \cap M_{w}$ has weight at least $\left(\frac{1}{2}-\delta\right) w\left(M_{w}\right)$ and, at the same time, the edge with minimum length in $C_{1} \cap M_{\ell}$ has length at least $\left(\frac{1}{2}-\delta\right) \ell\left(M_{\ell}\right)$. Since $1 / 2-\delta>1 / 3$, $C_{1}$ must be a cycle on four nodes, i.e. $C_{1} \cap M_{w}=\{(a, b),(c, d)\}$ and $C_{1} \cap M_{\ell}=\{(b, c),(a, d)\}$ (see Figure 2 for an illustration).

We deduce that $\max \{w(a, b), w(c, d)\}=w\left(M_{w}\right)-w\left(\bigcup_{i=2}^{p} C_{i} \cap M_{w}\right)-\min \{w(a, b), w(c, d)\}$ and $\max \{\ell(a, d), \ell(b, c)\}=\ell\left(M_{\ell}\right)-\ell\left(\bigcup_{i=2}^{p} C_{i} \cap M_{\ell}\right)-\min \{\ell(a, d), \ell(b, c)\}$. Using $\min \{w(a, b), w(c, d)\} \geq$ $(1 / 2-\delta) w\left(M_{w}\right)$ and $\min \{\ell(b, c), \ell(a, d)\} \geq(1 / 2-\delta) \ell\left(M_{\ell}\right)$ in the previous equalities gives

$$
\begin{aligned}
\max \{w(a, b), w(c, d)\} & \leq(1 / 2+\delta) w\left(M_{w}\right)-w\left(\bigcup_{i=2}^{p} C_{i} \cap M_{w}\right) \\
\max \{\ell(b, c), \ell(a, d)\} & \leq(1 / 2+\delta) \ell\left(M_{\ell}\right)-\ell\left(\bigcup_{i=2}^{p} C_{i} \cap M_{\ell}\right)
\end{aligned}
$$

In addition we deduce that

$$
w\left(\bigcup_{i=2}^{p} C_{i} \cap M_{w}\right) \leq 2 \delta w\left(M_{w}\right) \text { and } \ell\left(\bigcup_{i=2}^{p} C_{i} \cap M_{\ell}\right) \leq 2 \delta \ell\left(M_{\ell}\right)
$$

We conduct a subcase analysis depending on the weight or the length of the edges having at least one endpoint in $V\left(C_{1}\right)$ : case (1.1.w) $\max \left\{w(e): e \in C_{1} \cap M_{\ell}\right\}>2 \delta w\left(M_{w}\right)$, case (1.1. $)$ $\max \left\{\ell(e): e \in C_{1} \cap M_{w}\right\}>2 \delta \ell\left(M_{\ell}\right)$, case (1.2.w) $\max \{w(a, c), w(b, d)\}>\left(\frac{1}{2}+\delta\right) w\left(M_{w}\right)$, case (1.2. $\ell) \max \{\ell(a, c), \ell(b, d)\}>\left(\frac{1}{2}+\delta\right) \ell\left(M_{\ell}\right)$, case $(1.3 . w) \max \left\{w(i, j): i \in V\left(C_{1}\right), j \notin\right.$ $\left.V\left(C_{1}\right)\right\}>2 \delta w\left(M_{w}\right)$, case $(1.3 . \ell) \max \left\{\ell(i, j): i \in V\left(C_{1}\right), j \notin V\left(C_{1}\right)\right\}>2 \delta \ell\left(M_{\ell}\right)$ and case (1.4) $\max \left\{w(e): e \in C_{1} \cap M_{\ell}\right\} \leq 2 \delta w\left(M_{w}\right), \max \left\{\ell(e): e \in C_{1} \cap M_{w}\right\} \leq 2 \delta \ell\left(M_{\ell}\right)$, $\max \{w(a, c), w(b, d)\} \leq\left(\frac{1}{2}+\delta\right) w\left(M_{w}\right), \max \{\ell(a, c), \ell(b, d)\} \leq\left(\frac{1}{2}+\delta\right) \ell\left(M_{\ell}\right), \max \{w(i, j): i \in$ $\left.V\left(C_{1}\right), j \notin V\left(C_{1}\right)\right\} \leq 2 \delta w\left(M_{w}\right)$ and $\max \left\{\ell(i, j): i \in V\left(C_{1}\right), j \notin V\left(C_{1}\right)\right\} \leq 2 \delta \ell\left(M_{\ell}\right)$.

We can prove that in case (1.4) the instance $K_{n}$ satisfies $(i i)$ whereas in other cases the instance $K_{n}$ satisfies $(i)$. 
(1.1.w) If $w(a, d)>2 \delta w\left(M_{w}\right)$ or $w(b, c)>2 \delta w\left(M_{w}\right)$ then remove $(c, d)$. We get that $w(a, b)+$ $w(b, c)+w(a, d)>\left(\frac{1}{2}+\delta\right) w\left(M_{w}\right)$ and $\ell(a, b)+\ell(b, c)+\ell(a, d) \geq(1-2 \delta) \ell\left(M_{\ell}\right) \geq$ $(1 / 2+\delta) \ell\left(M_{\ell}\right)$

(1.1. $\ell)$ If $\ell(a, b)>2 \delta \ell\left(M_{\ell}\right)$ or $\ell(c, d)>2 \delta \ell\left(M_{\ell}\right)$ then remove $(b, c)$. We get that $\ell(a, d)+\ell(a, b)+$ $\ell(c, d)>\left(\frac{1}{2}+\delta\right) \ell\left(M_{\ell}\right)$ and $w(a, d)+w(a, b)+w(c, d) \geq(1-2 \delta) w\left(M_{w}\right) \geq(1 / 2+\delta) w\left(M_{w}\right)$.

(1.2.w) If $\max \{w(a, c), w(b, d)\}>\left(\frac{1}{2}+\delta\right) w\left(M_{w}\right)$ then remove $\{(a, b),(c, d)\}$ and add the edge with maximum weight between $(a, c)$ and $(b, d)$, say $(a, c)$ without loss of generality. We get that $w(a, c)+w(b, c)+w(a, d)>\left(\frac{1}{2}+\delta\right) w\left(M_{w}\right)$ and $\ell(a, d)+\ell(a, c)+\ell(b, c) \geq$ $(1-2 \delta) \ell\left(M_{\ell}\right) \geq(1 / 2+\delta) \ell\left(M_{\ell}\right)$.

(1.2. $\ell$ If $\max \{\ell(a, c), \ell(b, d)\}>\left(\frac{1}{2}+\delta\right) \ell\left(M_{\ell}\right)$ then remove $\{(a, d),(b, c)\}$ and add the edge with maximum length between $(a, c)$ and $(b, d)$, say $(a, c)$ without loss of generality. We get that $w(a, c)+w(a, b)+w(c, d)>(1-2 \delta) w\left(M_{w}\right)>(1 / 2+\delta) w\left(M_{w}\right)$ and $\ell(a, c)+\ell(a, b)+$ $\ell(c, d) \geq\left(\frac{1}{2}+\delta\right) \ell\left(M_{\ell}\right)$.

(1.3.w) Suppose there exists an edge $(i, j)$ such that $i \in\{a, b, c, d\}, j \in V \backslash\{a, b, c, d\}$ and $w(i, j)>2 \delta w\left(M_{w}\right)$. If $i \in\{a, b\}$ (resp. $\left.i \in\{c, d\}\right)$ then only keep the edges $\{(i, j),(b, c),(c, d),(a, d)\}$ (resp. $\{(i, j),(b, c),(a, b),(a, d)\})$ while any other edge is deleted. Suppose w.l.o.g. that $i \in\{a, b\}$, the case $i \in\{c, d\}$ being treated similarly. Using $w(c, d) \geq\left(\frac{1}{2}-\delta\right) w\left(M_{w}\right)$ and $\ell\left(C_{1} \cap M_{\ell}\right) \geq 2(1 / 2-\delta) \ell\left(M_{\ell}\right)$ by hypothesis, we get that $w(i, j)+w(b, c)+w(c, d)+$ $w(a, d) \geq w(i, j)+w(c, d)>(1 / 2+\delta) w\left(M_{w}\right)$. At the same time $\ell(i, j)+\ell(b, c)+\ell(c, d)+$ $\ell(a, d) \geq \ell(a, d)+\ell(b, c) \geq 2(1 / 2-\delta) \ell\left(M_{\ell}\right) \geq(1 / 2+\delta) \ell\left(M_{\ell}\right)$.

(1.3. $\ell$ ) Suppose there exists an edge $(i, j)$ such that $i \in\{a, b, c, d\}, j \in V \backslash\{a, b, c, d\}$ and $\ell(i, j)>$ $2 \delta \ell\left(M_{\ell}\right)$. If $i \in\{a, d\}$ (resp. $i \in\{b, c\}$ ) then only keep the edges $\{(i, j),(a, b),(b, c),(c, d)\}$ (resp. $\{(i, j),(a, b),(c, d),(a, d)\})$ while any other edge is deleted. Suppose w.l.o.g. that $i \in\{a, d\}$, the case $i \in\{b, c\}$ being treated similarly. Using $\ell(b, c) \geq\left(\frac{1}{2}-\delta\right) \ell\left(M_{\ell}\right)$ and $w\left(C_{1} \cap M_{w}\right) \geq 2(1 / 2-\delta) w\left(M_{w}\right)$ by hypothesis, we get that $\ell(i, j)+\ell(a, b)+\ell(b, c)+$ $\ell(c, d) \geq \ell(i, j)+\ell(b, c)>(1 / 2+\delta) \ell\left(M_{\ell}\right)$. At the same time $w(i, j)+w(a, b)+w(b, c)+$ $w(c, d) \geq w(a, b)+w(c, d) \geq 2(1 / 2-\delta) w\left(M_{w}\right) \geq(1 / 2+\delta) w\left(M_{w}\right)$.

(1.4) Suppose that $w(a, d) \leq 2 \delta w\left(M_{w}\right), w(b, c) \leq 2 \delta w\left(M_{w}\right), \ell(a, b) \leq 2 \delta \ell\left(M_{\ell}\right), \ell(c, d) \leq$ $2 \delta \ell\left(M_{\ell}\right), \max \{w(a, c), w(b, d)\} \leq\left(\frac{1}{2}+\delta\right) w\left(M_{w}\right)$ and $\max \{\ell(a, c), \ell(b, d)\} \leq\left(\frac{1}{2}+\delta\right) \ell\left(M_{\ell}\right)$. In addition suppose that for all $(i, j)$ such that $i \in\{a, b, c, d\}$ and $j \in V \backslash\{a, b, c, d\}$, we have $w(i, j) \leq 2 \delta w\left(M_{w}\right)$ and $\ell(i, j) \leq 2 \delta \ell\left(M_{\ell}\right)$. We claim that the weight of any tour is bounded above by $\left(\frac{3}{2}+7 \delta\right) w\left(M_{w}\right)$ while its length is at most $\left(\frac{3}{2}+7 \delta\right) \ell\left(M_{\ell}\right)$. The edge set of the graph is partitioned into three sets $E_{1}=\{(i, j): i, j \in\{a, b, c, d\}\}$, $E_{2}=\{(i, j): i \in\{a, b, c, d\}$ and $j \notin\{a, b, c, d\}\}$ and $E_{3}=\{(i, j): i, j \notin\{a, b, c, d\}\}$. A tour $T$ is a set of edges partitioned in three sets $T_{i}=T \cap E_{i}$ for $i=1,2,3$.

First observe that $T_{3}$ is a set of paths which can be decomposed into two matchings $M$ and $M^{\prime}$ (alternate edges in $M$ and edges in $M^{\prime}$ ). If $w(M) \geq w\left(M^{\prime}\right)$ and $w(M)>$ $w\left(\bigcup_{i=2}^{p} C_{i} \cap M_{w}\right)$ then $M \cup\{(a, b),(c, d)\}$ is a matching on the whole graph with larger weight than $w\left(M_{w}\right)$, contradiction. Using this argument and a similar one for the length we get that

$$
w\left(T_{3}\right) \leq 2 w\left(\bigcup_{i=2}^{p} C_{i} \cap M_{w}\right) \text { and } \ell\left(T_{3}\right) \leq 2 \ell\left(\bigcup_{i=2}^{p} C_{i} \cap M_{\ell}\right)
$$


We get that $w\left(C_{1} \cap M_{w}\right)=w\left(M_{w}\right)-w\left(\bigcup_{i=2}^{p} C_{i} \cap M_{w}\right)$ and $\ell\left(C_{1} \cap M_{\ell}\right)=\ell\left(M_{\ell}\right)-$ $\ell\left(\bigcup_{i=2}^{p} C_{i} \cap M_{\ell}\right)$. Thus, using inequality (7), we deduce that $w\left(C_{1} \cap M_{w}\right)+w\left(T_{3}\right) \leq$ $w\left(M_{w}\right)+w\left(\bigcup_{i=2}^{p} C_{i} \cap M_{w}\right)$ and $\ell\left(C_{1} \cap M_{\ell}\right)+\ell\left(T_{3}\right) \leq \ell\left(M_{\ell}\right)+\ell\left(\bigcup_{i=2}^{p} C_{i} \cap M_{\ell}\right)$; using inequality (6), we obtain

$$
w\left(C_{1} \cap M_{w}\right)+w\left(T_{3}\right) \leq(1+2 \delta) w\left(M_{w}\right) \text { and } \ell\left(C_{1} \cap M_{\ell}\right)+\ell\left(T_{3}\right) \leq(1+2 \delta) \ell\left(M_{\ell}\right)
$$

Because $\min \{w(a, b), w(c, d)\} \geq\left(\frac{1}{2}-\delta\right) w\left(M_{w}\right)$ and $\min \{\ell(b, c), \ell(a, d)\} \geq\left(\frac{1}{2}-\delta\right) \ell\left(M_{\ell}\right)$, we also deduce from inequality $(8)$ that:

$$
\begin{aligned}
\max \{w(a, b), w(c, d)\}+w\left(T_{3}\right) & \leq\left(\frac{1}{2}+3 \delta\right) w\left(M_{w}\right) \\
\max \{\ell(b, c), \ell(a, d)\}+\ell\left(T_{3}\right) & \leq\left(\frac{1}{2}+3 \delta\right) \ell\left(M_{\ell}\right)
\end{aligned}
$$

Since $M_{r}=\{(a, c),(b, d)\}$ is a matching on $V\left(C_{1}\right)$ (see Figure 2), we get that $w\left(M_{r}\right) \leq$ $w\left(C_{1} \cap M_{w}\right)$ and $\ell\left(M_{r}\right) \leq \ell\left(C_{1} \cap M_{\ell}\right)$. Thus, using inequality (8) we get that:

$$
w\left(M_{r}\right)+w\left(T_{3}\right) \leq(1+2 \delta) w\left(M_{w}\right) \text { and } \ell\left(M_{r}\right)+\ell\left(T_{3}\right) \leq(1+2 \delta) \ell\left(M_{\ell}\right)
$$

We also get that $w\left(M_{r}\right) \leq w\left(C_{1} \cap M_{w}\right)=w\left(M_{w}\right)-w\left(\bigcup_{i=2}^{p} C_{i} \cap M_{w}\right)$ and $\ell\left(M_{r}\right) \leq$ $\ell\left(C_{1} \cap M_{\ell}\right)=\ell\left(M_{\ell}\right)-\ell\left(\bigcup_{i=2}^{p} C_{i} \cap M_{\ell}\right)$. Thus, on the one hand, using inequalities (4) (resp., (5)) and (7), we deduce:

$$
\begin{aligned}
w\left(M_{r}\right)+\max \{w(a, b), w(c, d)\}+w\left(T_{3}\right) & \leq\left(\frac{3}{2}+\delta\right) w\left(M_{w}\right) \\
\ell\left(M_{r}\right)+\max \{\ell(b, c), \ell(a, d)\}+\ell\left(T_{3}\right) & \leq\left(\frac{3}{2}+\delta\right) \ell\left(M_{\ell}\right)
\end{aligned}
$$

Inequalities (7) and (6) also give

$$
w\left(T_{3}\right) \leq 4 \delta w\left(M_{w}\right) \text { and } \ell\left(T_{3}\right) \leq 4 \delta \ell\left(M_{\ell}\right)
$$

By hypothesis every edge in $E_{2}$ has weight (resp. length) at most $2 \delta w\left(M_{w}\right)$ (resp. $\left.2 \delta \ell\left(M_{\ell}\right)\right)$. It follows that

$$
w\left(T_{2}\right) \leq 2\left|T_{2}\right| \delta w\left(M_{w}\right) \text { and } \ell\left(T_{2}\right) \leq 2\left|T_{2}\right| \delta \ell\left(M_{\ell}\right)
$$

Now we argue on $T \cap E_{1}$. Note that $\left|T \cap E_{1}\right| \leq 3$ since $n \geq 5$. Then, if

- $T \cap E_{1}=\{(a, c),(b, d),(a, b)\}$. The tour must contain 2 edges in $E_{2}$. Thus, using inequalities (12) and (15) with $\left|T_{2}\right|=2$, we get $w(T)=w\left(M_{r}\right)+w(a, b)+w\left(T_{3}\right)+$ $w\left(T_{2}\right) \leq\left(\frac{3}{2}+\delta+4 \delta\right) w\left(M_{w}\right) \leq\left(\frac{3}{2}+7 \delta\right) w\left(M_{w}\right)$ and using inequality $(11) \ell(T)=$ $\ell\left(M_{r}\right)+\ell\left(T_{3}\right)+\ell(a, b)+\ell\left(T_{2}\right) \leq(1+2 \delta+2 \delta+4 \delta) \ell\left(M_{\ell}\right)=(1+8 \delta) \ell\left(M_{\ell}\right)<$ $\left(\frac{3}{2}+7 \delta\right) \ell\left(M_{\ell}\right)$. 
- $T \cap E_{1}=\{(a, c),(a, b),(c, d)\}$. The tour must contain 2 edges in $E_{2}$. Thus, using inequalities (8) and (15) with $\left|T_{2}\right|=2$, we get that $w(T)=w(a, c)+w\left(C_{1} \cap M_{w}\right)+$ $w\left(T_{3}\right)+w\left(T_{2}\right) \leq(1 / 2+\delta+1+2 \delta+4 \delta) w\left(M_{w}\right)=\left(\frac{3}{2}+7 \delta\right) w\left(M_{w}\right)$ and using inequality (14) $\ell(T)=\ell(a, c)+\ell(a, b)+\ell(c, d)+\ell\left(T_{2}\right)+\ell\left(T_{3}\right) \leq(1 / 2+\delta+2 \delta+2 \delta+4 \delta+$ $4 \delta) \ell\left(M_{\ell}\right)=(1 / 2+13 \delta) \ell\left(M_{\ell}\right)<\left(\frac{3}{2}+7 \delta\right) \ell\left(M_{\ell}\right)$.

$-T \cap E_{1}=\{(a, b),(b, c),(c, d)\}$. The tour must contain 2 edges in $E_{2}$. Thus, using inequalities (8) and (15) with $\left|T_{2}\right|=2$, we get that $w(T)=w\left(C_{1} \cap M_{w}\right)+w\left(T_{3}\right)+$ $w(b, c)+w\left(T_{2}\right) \leq(1+2 \delta+2 \delta+4 \delta) w\left(M_{w}\right)=(1+8 \delta) w\left(M_{w}\right)<\left(\frac{3}{2}+7 \delta\right) w\left(M_{w}\right)$. Using inequality (10) we get that $\ell(T)=\ell(a, b)+\ell(c, d)+\ell(b, c)+\ell\left(T_{2}\right)+\ell\left(T_{3}\right) \leq$ $(2 \delta+2 \delta+1 / 2+3 \delta+4 \delta) \ell\left(M_{\ell}\right)=(1 / 2+11 \delta)<\left(\frac{3}{2}+7 \delta\right) \ell\left(M_{\ell}\right)$

$-T \cap E_{1}=\{(a, c),(b, d)\}$. The tour must contain 4 edges in $E_{2}$. Thus, using inequalities (11) and (15) with $\left|T_{2}\right|=4, w(T)=w\left(M_{r}\right)+w\left(T_{3}\right)+w\left(T_{2}\right) \leq(1+2 \delta+$ $8 \delta) w\left(M_{w}\right)=(1+10 \delta) w\left(M_{w}\right)<\left(\frac{3}{2}+7 \delta\right) w\left(M_{w}\right)$ and $\ell(T)=\ell\left(M_{r}\right)+\ell\left(T_{3}\right)+\ell\left(T_{2}\right)=$ $(1+10 \delta) \ell\left(M_{\ell}\right) \leq\left(\frac{3}{2}+7 \delta\right) \ell\left(M_{\ell}\right)$.

$-T \cap E_{1}=\{(a, b),(a, c)\}$. The tour must contain 4 edges in $E_{2}$. Thus, using inequalities (9) and (15) with $\left|T_{2}\right|=4$, we get that $w(T)=w(a, b)+w\left(T_{3}\right)+$ $w(a, c)+w\left(T_{2}\right) \leq(1 / 2+3 \delta+1 / 2+\delta+8 \delta) w\left(M_{w}\right)=(1+12 \delta) w\left(M_{w}\right) \leq\left(\frac{3}{2}+7 \delta\right) w\left(M_{w}\right)$ and using inequality $(14) \ell(T)=\ell(a, b)+\ell(a, c)+\ell\left(T_{2}\right)+\ell\left(T_{3}\right) \leq(2 \delta+1 / 2+\delta+$ $8 \delta+4 \delta) \ell\left(M_{\ell}\right)=(1 / 2+15 \delta) \ell\left(M_{\ell}\right) \leq\left(\frac{3}{2}+7 \delta\right) \ell\left(M_{\ell}\right)$.

$-T \cap E_{1}=\{(a, b),(c, d)\}$. The tour must contain 4 edges in $E_{2}$. Thus, using inequalities (8) and (15) with $\left|T_{2}\right|=4, w(T)=w\left(C_{1} \cap M_{w}\right)+w\left(T_{3}\right)+w\left(T_{2}\right) \leq$ $(1+2 \delta+8 \delta) w\left(M_{w}\right)=(1+10 \delta) w\left(M_{w}\right) \leq\left(\frac{3}{2}+7 \delta\right) w\left(M_{w}\right)$ and using inequality (14) $\ell(T)=\ell(a, b)+\ell(c, d)+\ell\left(T_{2}\right)+\ell\left(T_{3}\right) \leq(2 \delta+2 \delta+8 \delta+4 \delta) \ell\left(M_{\ell}\right)=16 \delta \ell\left(M_{\ell}\right) \leq$ $\left(\frac{3}{2}+7 \delta\right) \ell\left(M_{\ell}\right)$.

$-T \cap E_{1}=\{(a, c)\}$. The tour must contain 6 edges in $E_{2}$. Thus, using inequalities (14) and (15) with $\left|T_{2}\right|=6, w(T)=w(a, c)+w\left(T_{2}\right)+w\left(T_{3}\right) \leq(1 / 2+\delta+12 \delta+$ $4 \delta) w\left(M_{w}\right)=(1 / 2+17 \delta) w\left(M_{w}\right) \leq\left(\frac{3}{2}+7 \delta\right) w\left(M_{w}\right)$ and $\ell(T)=\ell(a, c)+\ell\left(T_{2}\right)+$ $\ell\left(T_{3}\right) \leq(1 / 2+\delta+12 \delta+4 \delta) \ell\left(M_{\ell}\right)=(1 / 2+17 \delta) \ell\left(M_{\ell}\right) \leq\left(\frac{3}{2}+7 \delta\right) \ell\left(M_{\ell}\right)$.

$-T \cap E_{1}=\{(a, b)\}$. The tour must contain 6 edges in $E_{2}$. Thus, using inequalities (9) and (15) with $\left|T_{2}\right|=6, w(T)=w(a, b)+w\left(T_{3}\right)+w\left(T_{2}\right) \leq(1 / 2+3 \delta+12 \delta) w\left(M_{w}\right)=$ $(1 / 2+15 \delta) w\left(M_{w}\right) \leq\left(\frac{3}{2}+7 \delta\right) w\left(M_{w}\right)$ and using inequality $(14) \ell(T)=\ell(a, b)+$ $\ell\left(T_{2}\right)+\ell\left(T_{3}\right) \leq(2 \delta+12 \delta+4 \delta) \ell\left(M_{\ell}\right)=18 \delta \ell\left(M_{\ell}\right) \leq\left(\frac{3}{2}+7 \delta\right) \ell\left(M_{\ell}\right)$.

$-T \cap E_{1}=\emptyset$. To cover $a, b, c$ and $d$, the tour must contain 8 edges in $E_{2}$. Thus, using inequalities (14) and (15) with $\left|T_{2}\right|=8, w(T)=w\left(T_{2}\right)+w\left(T_{3}\right) \leq(16 \delta+$ $4 \delta) w\left(M_{w}\right)=20 \delta w\left(M_{w}\right) \leq\left(\frac{3}{2}+7 \delta\right) w\left(M_{w}\right)$ and $\ell(T)=\ell\left(T_{2}\right)+\ell\left(T_{3}\right) \leq(16 \delta+$ $4 \delta) \ell\left(M_{\ell}\right)=20 \delta \ell\left(M_{\ell}\right) \leq\left(\frac{3}{2}+7 \delta\right) \ell\left(M_{\ell}\right)$.

- Any other subcase is isomorphic to a previously analyzed subcase by flipping $w$ and $\ell$.

The conclusion is that every tour $T$ is such that $w(T) \leq\left(\frac{3}{2}+7 \delta\right) w\left(M_{w}\right)$ and $\ell(T) \leq$ $\left(\frac{3}{2}+7 \delta\right) \ell\left(M_{\ell}\right)$.

Case 2. Suppose that there exists a cycle, say $C_{1}$ w.l.o.g., such that the edge with minimum weight in $C_{1} \cap M_{w}$ has weight at most $\left(\frac{1}{2}-\delta\right) w\left(M_{w}\right)$ and, at the same time, the edge with minimum length in $C_{1} \cap M_{\ell}$ has length at least $\left(\frac{1}{2}-\delta\right) \ell\left(M_{\ell}\right)$. We will prove that the instance 
$K_{n}$ satisfies $(i)$. Again, since $1 / 2-\delta>1 / 3, C_{1}$ must be a cycle on four nodes. Again we suppose that $C_{1} \cap M_{w}=\{(a, b),(c, d)\}$ and $C_{1} \cap M_{\ell}=\{(b, c),(a, d)\}$.

Remove the edge in $C_{1} \cap M_{w}$ with minimum weight and for any other cycle $C_{i}$ remove one edge in $C_{i} \cap M_{\ell}$ arbitrarily. We get a partial tour. Since $w\left(M_{w}\right)-\min \{w(a, b), w(c, d)\} \geq$ $\left(\frac{1}{2}+\delta\right) w\left(M_{w}\right)$ and $\ell\left(C_{1} \cap M_{\ell}\right)=\ell(a, d)+\ell(b, c) \geq 2\left(\frac{1}{2}-\delta\right) \ell\left(M_{\ell}\right) \geq\left(\frac{1}{2}+\delta\right) \ell\left(M_{\ell}\right)$, the partial tour has weight (resp. length) at least $\left(\frac{1}{2}+\delta\right) w\left(M_{w}\right)$ (resp. $\left.\left(\frac{1}{2}+\delta\right) \ell\left(M_{\ell}\right)\right)$.

The case where there exists a cycle $C_{1}$ such that the edge with minimum weight in $C_{1} \cap M_{w}$ has weight at least $\left(\frac{1}{2}-\delta\right) w\left(M_{w}\right)$ and, at the same time, the edge with minimum length in $C_{1} \cap M_{\ell}$ has length at most $\left(\frac{1}{2}-\delta\right) \ell\left(M_{\ell}\right)$ is dealt with similar arguments by flipping $w$ and $\ell$.

Case 3. Denote by $e_{i}^{w}$ (resp. $e_{i}^{\ell}$ ) the edge in $C_{i} \cap M_{w}$ (resp. $C_{i} \cap M_{\ell}$ ) with minimum weight (resp. length). We deal with the remaining case where $w\left(e_{i}^{w}\right) \leq\left(\frac{1}{2}-\delta\right) w\left(M_{w}\right)$ and $\ell\left(e_{i}^{\ell}\right) \leq\left(\frac{1}{2}-\delta\right) \ell\left(M_{\ell}\right)$ for all $i \in\{1, \ldots, p\}$. We will prove that the instance $K_{n}$ satisfies $(i)$. Since every cycle contains at least two edges of $M_{w}$ and also two edges of $M_{\ell}$ we deduce that

$$
\sum_{i=1}^{p} w\left(e_{i}^{w}\right) \leq w\left(M_{w}\right) / 2 \text { and } \sum_{i=1}^{p} \ell\left(e_{i}^{\ell}\right) \leq \ell\left(M_{\ell}\right) / 2
$$

- Suppose there is an index $i^{*}$ such that $w\left(e_{i^{*}}^{w}\right) \geq \delta w\left(M_{w}\right)$. Then for every cycle $C_{i}$ except $C_{i^{*}}$ remove $e_{i}^{w}$. Remove $e_{i^{*}}^{\ell}$. Using the first part of inequality (16) we get a partial tour with weight at least $w\left(M_{w}\right)-\sum_{i=1}^{p} w\left(e_{i}^{w}\right)+w\left(e_{i^{*}}^{w}\right) \geq(1 / 2+\delta) w\left(M_{w}\right)$ and length at least $\ell\left(M_{\ell}\right)-\ell\left(e_{i^{*}}^{\ell}\right) \geq(1 / 2+\delta) \ell\left(M_{\ell}\right)$.

- Suppose there is an index $i^{*}$ such that $\ell\left(e_{i^{*}}^{\ell}\right) \geq \delta \ell\left(M_{\ell}\right)$. With similar arguments we can build a partial tour with weight at least $(1 / 2+\delta) w\left(M_{w}\right)$ and length at least $(1 / 2+$ $\delta) \ell\left(M_{\ell}\right)$.

- Suppose that $w\left(e_{i}^{w}\right)<\delta w\left(M_{w}\right)$ and $\ell\left(e_{i}^{\ell}\right)<\delta \ell\left(M_{\ell}\right)$ for all $i$. If $\sum_{i=1}^{p} w\left(e_{i}^{w}\right) \leq\left(\frac{1}{2}-\right.$ $\delta) w\left(M_{w}\right)$, then by removing $e_{i}^{w}$ for $i=1, \ldots, p$ we get a partial tour $P$ with weight at least $(1 / 2+\delta) w\left(M_{w}\right)$ and length at least $\ell\left(M_{\ell}\right)$. Otherwise, there exists an index $i^{*}<p$ such that

$$
\sum_{i=1}^{i^{*}} w\left(e_{i}^{w}\right) \leq\left(\frac{1}{2}-\delta\right) w\left(M_{w}\right) \text { and } \sum_{i=1}^{i^{*}+1} w\left(e_{i}^{w}\right)>\left(\frac{1}{2}-\delta\right) w\left(M_{w}\right)
$$

Using inequalities (16), (17) and $w\left(e_{i^{*}+1}^{w}\right)<\delta w\left(M_{w}\right)$ we get that

$$
\begin{aligned}
\sum_{i=1}^{i^{*}+1} w\left(e_{i}^{w}\right)+\sum_{i=i^{*}+2}^{p} w\left(e_{i}^{w}\right) & \leq w\left(M_{w}\right) / 2 \\
\sum_{i=i^{*}+2}^{p} w\left(e_{i}^{w}\right) & <\delta w\left(M_{w}\right) \\
\sum_{i=i^{*}+1}^{p} w\left(e_{i}^{w}\right) & <2 \delta w\left(M_{w}\right) \leq\left(\frac{1}{2}-\delta\right) w\left(M_{w}\right)
\end{aligned}
$$


Now remark that

$$
\min \left\{\sum_{i=1}^{i^{*}} \ell\left(e_{i}^{\ell}\right), \sum_{i=i^{*}+1}^{p} \ell\left(e_{i}^{\ell}\right)\right\} \leq \frac{1}{2} \sum_{i=1}^{p} \ell\left(e_{i}^{\ell}\right) \leq \frac{1}{4} \ell\left(M_{\ell}\right)
$$

where the right part of inequality (16) is used. If $\sum_{i=1}^{i^{*}} \ell\left(e_{i}^{\ell}\right) \leq \sum_{i=i^{*}+1}^{p} \ell\left(e_{i}^{\ell}\right)$ then remove $e_{i}^{\ell}$ for $i=1, \ldots, i^{*}$ and remove $e_{i}^{w}$ for $i=i^{*}+1, \ldots, p$. We get a partial tour with weight at least $(1 / 2+\delta) w\left(M_{w}\right)$ by inequality (18) and length at least $3 \ell\left(M_{\ell}\right) / 4 \geq(1 / 2+\delta) \ell\left(M_{\ell}\right)$ by inequality (19). If $\sum_{i=1}^{i^{*}} \ell\left(e_{i}^{\ell}\right)>\sum_{i=i^{*}+1}^{p} \ell\left(e_{i}^{\ell}\right)$ then remove $e_{i}^{w}$ for $i=1, \ldots, i^{*}$ and remove $e_{i}^{\ell}$ for $i=i^{*}+1, \ldots, p$. We get a partial tour with weight at least $(1 / 2+\delta) w\left(M_{w}\right)$ by inequality (17) and length at least $3 \ell\left(M_{\ell}\right) / 4 \geq(1 / 2+\delta) \ell\left(M_{\ell}\right)$ by inequality (19).

Acknowledgement: We thank Bruno Escoffier for stimulating discussions on the topic and an anonymous referee for his valuable comments on the first version.

\section{References}

[1] Eric Angel, Evripidis Bampis, and Aleksei V. Fishkin. A note on scheduling to meet two min-sum objectives. Operations Research Letters, 35(1):69-73, 2007.

[2] Eric Angel, Evripidis Bampis, and Laurent Gourvès. Approximating the Pareto curve with local search for the bicriteria TSP $(1,2)$ problem. Theoretical Computer Science, 310(1-3):135-146, 2004.

[3] Eric Angel, Evripidis Bampis, and Laurent Gourvès. Approximation algorithms for the bi-criteria weighted max-cut problem. Discrete Applied Mathematics, 154(12):1685-1692, 2006.

[4] Eric Angel, Evripidis Bampis, Laurent Gourvès, and Jérôme Monnot. (Non)approximability for the multi-criteria TSP $(1,2)$. In Proceeding of the 15 th International Symposium on Fundamentals of Computation Theory (FCT 2005), LNCS 3623, pages 329-340, 2005.

[5] Eric Angel, Evripidis Bampis, and Alexander Kononov. On the approximate tradeoff for bicriteria batching and parallel machine scheduling problems. Theoretical Computer Science, 306(1-3):319-338, 2003.

[6] André Berger, Vincenzo Bonifaci, Fabrizio Grandoni, and Guido Schäfer. Budgeted matching and budgeted matroid intersection via the gasoline puzzle. Mathematical Programming, 128(1-2):355-372, 2011.

[7] Markus Bläser, Bodo Manthey, and Oliver Putz. Approximating multi-criteria Max-TSP. In Proceedings of the 16th Annual European Symposium (ESA 2008), LNCS 5193, pages 185-197, 2008.

[8] Matthias Ehrgott. Approximation algorithms for combinatorial multicriteria optimization problems. International Transactions in Operational Research, 7:2000, 2000.

[9] Matthias Ehrgott. Multicriteria optimization. LNEMS, Springer-Verlag, 2005. 
[10] Thomas Erlebach, Hans Kellerer, and Ulrich Pferschy. Approximating multiobjective knapsack problems. Management Science, 48(12):1603-1612, 2002.

[11] Christian Glaßer, Christian Reitwießner, and Maximilian Witek. Applications of discrepancy theory in multiobjective approximation. In Supratik Chakraborty and Amit Kumar, editors, FSTTCS, volume 13 of LIPIcs, pages 55-65. Schloss Dagstuhl - Leibniz-Zentrum fuer Informatik, 2011.

[12] Sung-Pil Hong, Sung-Jin Chung, and Bum Hwan Park. A fully polynomial bicriteria approximation scheme for the constrained spanning tree problem. Operations Research Letters, 32(3):233-239, 2004.

[13] Lukasz Kowalik and Marcin Mucha. Deterministic 7/8-approximation for the metric maximum TSP. Theoretical Computer Science, 410(47-49):5000-5009, 2009.

[14] Bodo Manthey. Deterministic algorithms for multi-criteria Max-TSP. Discrete Applied Mathematics, 160(15):2277-2285, 2012.

[15] Bodo Manthey. Multi-criteria TSP: Min and max combined. Oper. Res. Lett., 40(1):36$38,2012$.

[16] Bodo Manthey. On approximating multicriteria TSP. ACM Transactions on Algorithms, 8(2):17, 2012.

[17] Bodo Manthey and L. Shankar Ram. Approximation algorithms for multi-criteria Traveling Salesman Problems. Algorithmica, 53(1):69-88, 2009.

[18] Katarzyna E. Paluch, Marcin Mucha, and Aleksander Madry. A 7/9 - approximation algorithm for the maximum traveling salesman problem. In Proceedings of the 12th International Workshop on Approximation, Randomization, and Combinatorial Optimization. Algorithms and Techniques (APPROX-RANDOM 2009), LNCS 5687, pages 298-311, 2009.

[19] Christos H. Papadimitriou and Mihalis Yannakakis. On the approximability of trade-offs and optimal access of web sources. In Proceedings of the 41st Annual Symposium on Foundations of Computer Science (FOCS 2000), pages 86-92, 2000.

[20] April Rasala, Clifford Stein, Eric Torng, and Patchrawat Uthaisombut. Existence theorems, lower bounds and algorithms for scheduling to meet two objectives. In Proceedings of the 13th Annual ACM-SIAM Symposium on Discrete Algorithms (SODA 2002), pages 723-731, 2002.

[21] R. Ravi and Michel X. Goemans. The constrained minimum spanning tree problem. In Proceeding of the 5th Scandinavian Workshop on Algorithm Theory (SWAT 1996), LNCS 1097, pages 66-75, 1996.

[22] R. Ravi, Madhav V. Marathe, S. S. Ravi, Daniel J. Rosenkrantz, and Harry B. Hunt III. Many birds with one stone: multi-objective approximation algorithms. In Proceedings of the 23rd Annual ACM Symposium on Theory of Computing (STOC 1993), pages 438-447, 1993. 
[23] Clifford Stein and Joel Wein. On the existence of schedules that are near-optimal for both makespan and total weighted completion time. Operations Research Letters, 21(3):115$122,1997$. 